\title{
Principle and Method of Image Recognition under Diffusive Distortions of Image
}

\author{
Jaser Doroshenko ${ }^{1}$, Lev Dulkin ${ }^{1}$, Viktor Salakhutdinov ${ }^{2}$, and Yury Smetanin ${ }^{3}$ \\ 1 Central Clinical Hospital of the Russian Academy of Sciences, Litovsky boul. 1a, \\ 119333, Moscow, Russia \\ retinaretina.ru
}

2 Research Institute of System Investigations of the Russian Academy of Sciences, Nahimovsky av.36-1, 117218, Moscow, Russia

vsalakhutdinovgmail.com

3 Computer Center of the Russian Academy of Sciences, Vavilov st. 40, 119333,

Moscow, Russia

smetaninrfbr.ru

\begin{abstract}
The results of investigation and development of software and hardware tools for processing images of ring objects under diffusive dissipation are presented. The proposed algorithm was demonstrated to be efficient in endoscopy for measuring the sizes of biological objects. A principal scheme of a hardware system for the implementation of the new method is presented. A plan of clinical testing of the new system is developed.
\end{abstract}

Keywords: evidence-based medicine, biomedical informatics, image recognition, endoscope, measurement algorithm.

\section{Introduction}

One of the most important topics in evidence-based medicine is the objective evaluation of the quality of clinical research. Practice demonstrates that many contemporary methods of measuring do not meet the requirements of evidencebased medicine. That is especially true for medical image processing and analysis.

The importance of endoscopic images in medicine is the consequence of the fact that now they are the main, and sometimes the only possible source of information for noninvasive visualization of internal anomalies and for the diagnostics of various diseases [1. The implementation of the endoscopic imaging is based on small size TV systems that are inserted into the investigated region. If the size is large, mosaicking is necessary.

The most important deadlock is that modern endoscopic methods and systems do not measure the distance between the camera and the object, therefore, it is impossible to match visible sizes of the objects in the image to their real size. The error in the estimation of the distance inevitably causes a wrong estimation of the size of the pathology, and as a consequence, a wrong strategic selection of treatment. In practice, the error of measurement in traditional endoscopic 
methods in some cases (e.g., in urologic checkup) is up to 3 to 5 times higher than it is needed for the selection of the adequate strategy of treatment [4. It reduces the efficiency of endoscopic monitoring and diagnostics in TV medicine.

Below, we consider an approach to solving this problem that is based on scaling using test images with a priori known sizes. A light spot is projected onto the image of the object. The size of the projection is used for the estimation of the scaling factor of the pathologies. The approach is well known and extensively used [56], but is precision is limited. In all practical implementations of the approach, difficulties appear that are related to great aberrations of the optical system of the endoscope [7]. Moreover, light dispersion occurs in a biological tissue which results in blurring and incorrect scaling.

A new system is proposed for imaging and processing. The system makes it possible to evaluate the real size of the pathologies using their endoscopic images. It is also possible to evaluate the errors caused by the light dispersion and to correct them.

\section{Principle and Methods}

The measuring system consists of a PC, TV endoscope whose brightening subsystem uses an inertia-free light-emitting diode, and light fibers. At one end, a laser light source is situated, at the other, micro collimator, which gives at the output a light beam whose section is equal along the whole length.

The light fiber is situated in the instrumental channel of the endoscope in such a way that it is possible to measure the direction of the laser beam by standard manipulators fixed at the distal end of the endoscope. This solution makes it possible to use optical systems with various angles between their optical axis and the axis of the instrumental channel (Fig. 1).

Microcollimator situated at the end of the light fiber transforms the divergent light beam into the beam with constant section. The PC controls the lightemitting diode used as the light source, the laser at the input of the light fiber, and the image processing for the TV endoscope.

The object whose size is measured is placed near the center of the image. After receiving the signal:

1. A current image of the object near the center is registered in the operating memory and displayed at the monitor as a static frame.

2. Along the foreground of the pulse of the frame synchronization for the TV endoscope, the light-diode source at the input of the light fiber is switched on.

3. The next frame, which consists of the image of the projection of the collimated laser beam light onto the measured object, is registered in the operating memory.

4. As the result of the image processing (step 3), the section of the laser beam is quantified (in pixels).

5. In the static image, a line is manually marked whose size is to be measured. The length of the line is defined as its size in pixels divided by the size in pixels 


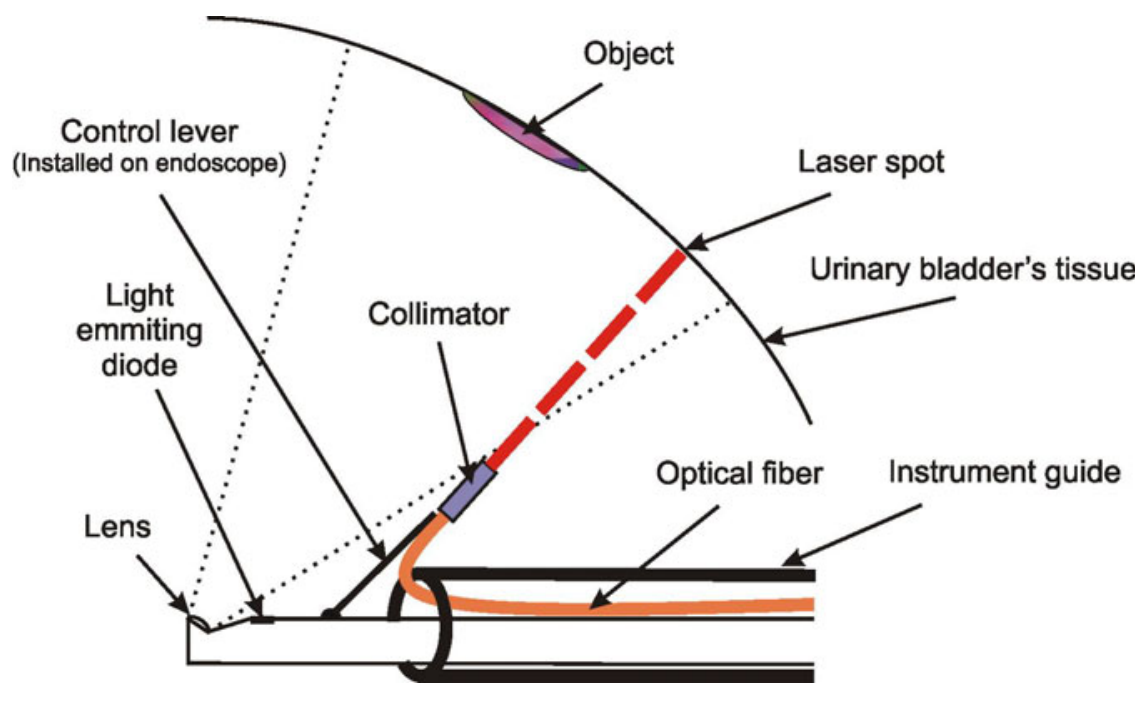

Fig. 1. The principal scheme of the distal end of the endoscope
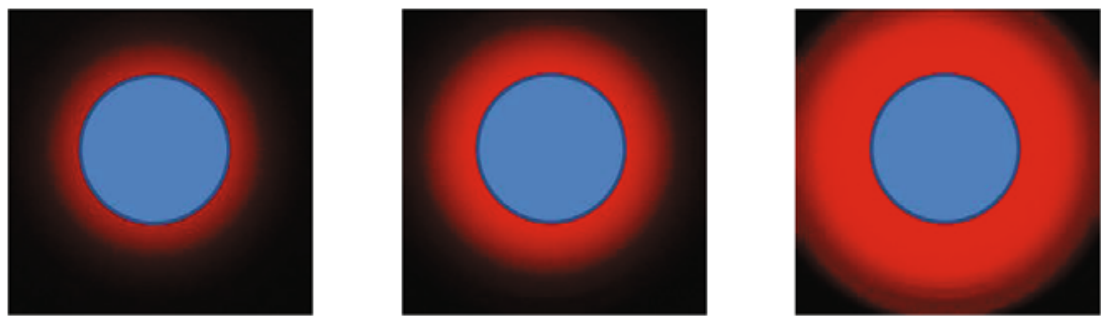

Fig. 2. Various halos around the laser beam

of the section of the laser beam and multiplied by the section of the laser beam in millimeters; the latter is known a priori.

\section{Calibration Using Circular Spots}

The considered problem is not trivial because, depending on the characteristics of the tissue of the measured object, the laser beam can either penetrate or not penetrate into the object, and the penetration may be up to the considerable depth. It may result in a halo whose intensity is comparable to the intensity of the main beam (Fig. 2, the blue circle is the initial laser spot) and, as a consequence, considerable errors of measurements appear The results of clinical testing demonstrate that the error caused by the dispersion may reach $70 \%$ in the processing of real images in medical endoscopy [8]. 

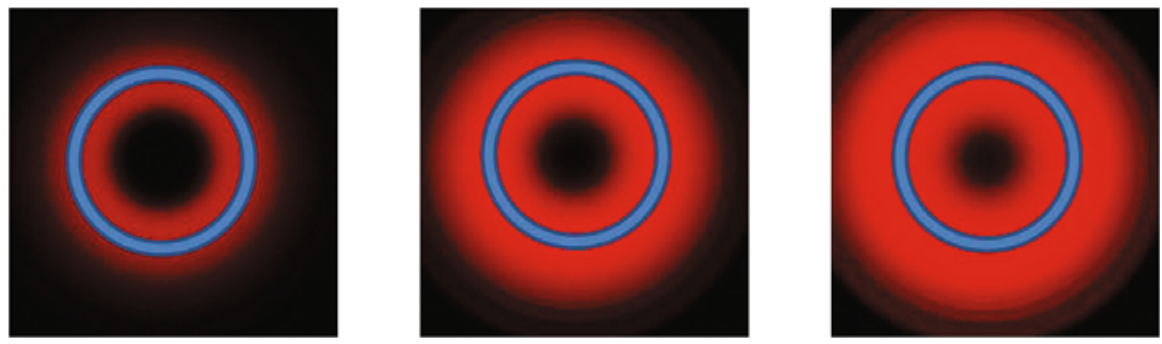

Fig. 3. Various halos around the laser beam that has the form of a circle

In order to enhance the precision, the laser beam at the output of the collimator has the form of the circle. In the process of measuring, the size of the projection of the laser beam onto the measured object (step 4), the measured value of the intensity in the center is used as the threshold. In Fig. 3, the dissipation of the circular laser beam is demonstrated.

Evidently, the diameter of the circle, which is clearly discernible at the images, is almost the same for all the values of the dispersion.

Clinical tests have demonstrated that eliminating the dispersion in the tissue reduces the error of measurements approximately by a factor of three, which is sufficient for practical medicine. The experiments with laboratory animals have demonstrated that the proposed system enhances the precision more than by an order of magnitude; that results in the substantially reduced fraction of incorrect diagnostic decisions.

The next step of image processing is the elimination of disturbances caused by the inhomogeneity of optical properties of biological tissue. The aim of this step is to obtain regular circles.

\section{Thresholds for the Determination of Regular Circles}

The proposed method is based on the model of optical coherence tomography of heterogeneous tissue [9] and the principles of the diffusion wave spectroscopy in inhomogeneous flows [10].

The transport equation is

$$
\frac{1}{c} \frac{\partial I(r, s, t)}{\partial t}+\nabla I(r, s, t) s+\mu_{t} I(r, s, t)=\mu_{s} \int I(r, s, t) p\left(s, s^{\prime}\right) d s^{\prime}+S(r, s, t),
$$

where $c$ is the velocity of light in the environment and $S(r, s, t)$ is the spatial and angular distribution of sources.

To cope with the complexity of this integro-differential equation, the expansion in series of spherical harmonics $Y_{l, m}$ is used, which results in a system of $(N+1)^{2}$ coupled partial differential equations. Thus we obtain 


$$
\begin{aligned}
& I(r, s, t)=\sum_{l=0}^{m} \sum_{m=-1}^{l} \psi_{l, m} Y_{i, m}(s) \\
& S(r, s, t)=\sum_{l=0}^{m} \sum_{m=-1}^{l} q_{l, m} Y_{i, m}(s)
\end{aligned}
$$

and in $P_{1}$ approximation,

$$
I(r, s, t)=U(r, t)+\frac{3}{4 \pi} F(r, t) s,
$$

where

$$
F(r, t)=\int_{4 \pi} I(r, s, t) s d s .
$$

In practice, it is sufficient to take the intensity in the center of the light spot multiplied by a const. $>1$. The result is used as the threshold to deduct from the signal. The exact value of the const. is defined by the quality of the input image and the required reliability.

\section{Conclusion}

The presented data demonstrate that the proposed method of measuring and reconstructing gives images with high quality topological structure, which are convenient for the interpretation. The system is easy to implement since it does not demand any complex hardware. The system is convenient for users that have no experience in computer technologies. It does not demand any changes in the optical system; it is important in the considered domain, where stable spectral and temporal characteristics, small thermal effect, etc. are required. After solving the problems of safety, which are inevitable in medical devices, the new system may be used as a subsystem of hardware and software tools for noninvasive registration not demanding contrast agents, localization of objects and regions, and monitoring of zones of abnormal blood supply of fundus. A plan of clinical testing of the new system is developed.

Acknowledgments. The work was partly supported by Russian Foundation for Basic Research, grants no. 09-07-00309-a, 09-07-00444-a, and 09-08-00993-a.

\section{References}

1. Cotton, P.B., Williams, C.B.: Practical Gastrointestinal Endoscopy: The Fundamentals, vol. 1. Blackwell Science, Oxford (2003)

2. McPhee, S.J., Papadakis, M.A.: Current Medical Diagnosis and Treatment 2009, vol. 1. McGraw Hill, New York (2009)

3. Sokolov, B.A., et al.: Endoscopic tactics in concretions that are hard to remove (in Russian). In: 9th Moscow International Congress on Endoscopic Surgery, Moscow, April 6 - 8, 2005, pp. 353-355. 
4. Dul'kin, L.M., Salakhutdinov, V.K., et al.: Method of longitudinal stereoscopy for $3 \mathrm{D}$ visualization of endoscopic images (in Russian). In: Proc. of Int. Symp. Topical Problems of Biophotonics-2007, pp. 1-30 (2007)

5. Horn, B.K.P.: Robot Vision, vol. 1. MIT Press, Cambridge (1986)

6. Kuriksha, Zhulina, Y.V.: Processing of corrupted optical images (in Russian). Radiotekhnika i elektronila 66(3), 23-40 (2000)

7. Born, M., Wolf, E.: Principles of Optics. Cambridge University Press, Cambridge (1999)

8. Dul'kin, L.M., et al.: Classification of complexity categories of diagnostical and clinical endoscopic retrograde pancreatocholangiography and the degree of risk of complications. In: 6th Moscow International Congress on Endoscopic Surgery, Moscow (2002)

9. Schmitt, J.M., Knutel, A.: Model of optical coherence tomography of heterogeneous tissue. J. Opt. Soc. Am. A. 14, 1231-1242 (1997)

10. Bicout, D., Maynard, R.: Diffusion wave spectroscopy in inhomogeneous flows. Physica A 199(3-4), 387-411 (1993) 